\title{
Large-scale RNAi screens identify novel genes that interact with the C. elegans retinoblastoma pathway as well as splicing-related components with synMuv B activity
}

\author{
Julian Ceron ${ }^{1,4}$, Jean-François Rual2 ${ }^{2}$ Abha Chandra1, Denis Dupuy², \\ Marc Vidal ${ }^{2}$ and Sander van den Heuvel*1,3
}

\begin{abstract}
Address: ${ }^{1}$ Massachusetts General Hospital Cancer Center and Harvard Medical School, Building 149, 13th Street, Charlestown, 02129 MA, USA, ${ }^{2}$ Center for Cancer Systems Biology (CCSB) and Department of Cancer Biology, Dana-Farber Cancer Institute, and Department of Genetics, Harvard Medical School, 44 Binney Street, Boston, 02115 MA, USA, ${ }^{3}$ Department of Developmental Biology, Utrecht University, Kruytbuilding, Padualaan 8, $3584 \mathrm{CH}$ Utrecht, The Netherlands and ${ }^{4}$ Present address : Molecular Oncology and Aging Research, Centre d'Investigacions en Bioquímica I Biología Molecular (CIBBIM), Hospital Universitari Vall d'Hebron 119-129, Barcelona 08035, Spain
\end{abstract}

Email: Julian Ceron - jceron@ir.vhebron.net; Jean-François Rual - JRUAL@partners.org; Abha Chandra - abha_6@hotmail.com; Denis Dupuy - denis_dupuy@dfci.harvard.edu; Marc Vidal - marc_vidal@dfci.harvard.edu; Sander van den

Heuvel* - S.J.L.vandenheuvel@bio.uu.nl

* Corresponding author

Published: 6 April 2007

BMC Developmental Biology 2007, 7:30 doi:10.1186/147/-213X-7-30

This article is available from: http://www.biomedcentral.com/I47/-2/3X/7/30

(C) 2007 Ceron et al; licensee BioMed Central Ltd.

This is an Open Access article distributed under the terms of the Creative Commons Attribution License (http://creativecommons.org/licenses/by/2.0), which permits unrestricted use, distribution, and reproduction in any medium, provided the original work is properly cited.
Received: 25 March 2007

Accepted: 6 April 2007

\begin{abstract}
Background: The retinoblastoma tumor suppressor $(\mathrm{Rb})$ acts in a conserved pathway that is deregulated in most human cancers. Inactivation of the single Rb-related gene in Caenorhabditis elegans, lin-35, has only limited effects on viability and fertility, yet causes changes in cell-fate and cell-cycle regulation when combined with inactivation of specific other genes. For instance, lin-35 $\mathrm{Rb}$ is a synthetic multivulva (synMuv) class $B$ gene, which causes a multivulva phenotype when inactivated simultaneously with a class $A$ or $C$ synMuv gene.
\end{abstract}

Results: We used the ORFeome RNAi library to identify genes that interact with C. elegans lin-35 $\mathrm{Rb}$ and identified 57 genes that showed synthetic or enhanced RNAi phenotypes in lin-35 mutants as compared to rrf-3 and eri-I RNAi hypersensitive mutants. Based on characterizations of a deletion allele, the synthetic lin-35 interactor $z f p-2$ was found to suppress RNAi and to cooperate with lin-35 Rb in somatic gonad development. Interestingly, ten splicing-related genes were found to function similar to lin-35 Rb, as synMuv $B$ genes that prevent inappropriate vulval induction. Partial inactivation of specific spliceosome components revealed further similarities with lin-35 Rb functions in cell-cycle control, transgene expression and restricted expression of germline granules.

Conclusion: We identified an extensive series of candidate lin-35 Rb interacting genes and validated $z f p-2$ as a novel lin-35 synthetic lethal gene. In addition, we observed a novel role for a subset of splicing components in lin-35 Rb-controlled processes. Our data support novel hypotheses about possibilities for anti-cancer therapies and multilevel regulation of gene expression. 


\section{Background}

The retinoblastoma gene $(\mathrm{Rb})$ was the first tumor suppressor gene to be genetically identified and cloned, based on germline mutations in familial cases of retinoblastoma [1]. Since, deregulation of the Rb pathway has been found to be a general aspect of a wide variety of human cancers, with $\sim 80 \%$ of all sporadic cancers containing alterations in $\mathrm{Rb}$ or its regulatory components $[2,3]$. The frequency of mutations in the $\mathrm{Rb}$ gene itself varies greatly between different tumor types, which likely relates to expression levels of other members of the Rb family (p130 and p107) that are closely related in structure and show partly redundant functions in vivo [4].

Proteins of the $\mathrm{pRb}$ family function as transcriptional repressors to control cell division, differentiation and death [[5], and citations herein]. To control cell proliferation, $\mathrm{pRb}$ family members inhibit transcription of $\mathrm{S}$ phase promoting genes by binding and blocking activating E2F transcription factors (in mammals: E2F-1, E2F-2 or E2F-3 in association with DP-1 or DP-2). Another group of E2F transcription factors acts together with the pRb protein family to repress transcription, including heterodimers of mammalian E2F-4 or E2F-5 and one of the two DP proteins [6]. Besides E2F family members, pRb-related proteins have been reported to physically interact with a large number of other cellular proteins, including components of chromatin remodeling complexes [e.g.: $[7,8]]$. Although the physiological relevance of many interactions remains to be determined, the functions of $\mathrm{pRb}$ likely involve a variety of different protein complexes that either block activation of transcription, or actively repress transcription through recruitment of histone-modification and chromatin-remodeling complexes.

The gene lin-35 encodes the single Caenorhabditis elegans homolog of the pRb family. Animals with putative null mutations in lin-35 are viable and fertile [9], although homozygous mutants show a reduced brood size, delayed development, and larval arrest with low penetrance $[[10,11]$ and our unpublished observations]. Previous studies revealed redundant functions of lin-35 in a variety of processes such as vulva formation, cell-cycle control, development of the pharynx and somatic gonad, larval development, distal tip cell (DTC) migration and meiotic progression [9-21].

The first and best-characterized function of lin-35 is inhibition of the vulval cell fate [[9] and references herein]. In this process, lin-35 acts as a synthetic Multivulva (synMuv) class B gene redundantly with class $\mathrm{A}$ and $\mathrm{C}$ genes [20]. Only animals that contain mutations in two different classes, e.g. A and B, display a Multivulva phenotype (a phenotype that depends on two simultaneous mutations is referred to as "synthetic", hence synMuv). Several
synMuv B genes act also in cell-cycle control [12,22] and many encode homologs of well-known partners of $\mathrm{pRb}$, including efl-1 E2F and $d p l-1$ DP [23]. Biochemical purification of $\mathrm{Rb}$ complexes revealed extensive overlap between components of the Drosophila myb/dREAM complex and C. elegans synMuv B proteins $[6,24]$. Together, these data highlight the evolutionary conservation of the $\mathrm{Rb}$ pathway as well as the valuable contributions of synMuv B mutants in identifying functional partners of $\mathrm{Rb}$ family members.

Since lin-35 is a non-essential gene and its functions are redundant, we decided to perform a synthetic screen to identify additional genes that interact with $l i n-35 \mathrm{Rb}$. This screen aimed to identify genes whose functions are essential specifically when lin-35 Rb is inactive. As the Rb pathway is generally compromised in tumor cells, this strategy has the potential of identifying targets for anticancer drugs, because inactivating a gene that is synthetic lethal with loss of $\mathrm{Rb}$ activity could preferentially reduce the viability of cancer cells. In a large-scale RNAi approach based on the ORFeome RNAi library [25], we identified 57 lin35 interacting genes. In addition, we found a novel group of synMuv B genes that encode components of the splicing machinery. Thus, our screens have revealed many novel genes that may act in parallel or in a linear pathway with lin-35 Rb, some of which may be followed as candidate targets for cancer therapies.

\section{Results and discussion}

To expand on previous studies of synthetic interactions with lin-35 Rb, we used RNAi by feeding to inhibit the function of 10,953 genes present in the ORFeome RNAi library v1.1. We compared the effects of feeding RNAi in wild-type (WT) animals and lin-35(n2239) Rb candidate "null" mutants [9]. In the initial screen, we identified 523 genes that showed a variety of synthetic or enhanced phenotypes in lin-35(n2239) as compared to WT (N2) animals (see: Methods; Additional file 1, Supplementary Table 1A; for legend, see Additional file 6).

lin-35 $\mathrm{Rb}$ and several other genes that act with $\mathrm{Rb}$ in transcriptional repression and chromatin remodeling in other species have been identified in C. elegans based on their inhibitory role in vulval cell-fate specification. Several strains deficient for these so-called synMuv B genes, including lin-35 mutants, were recently found to be RNAi hypersensitive [26]. To exclude candidates that show a non-specific stronger RNAi phenotype in lin-35(n2239), we included the non- $\mathrm{Rb}$ related RNAi hypersensitive strain $r r f-3$ (pk1426) in a secondary screen [27]. This assay removed 279 initially selected genes, as they showed similar RNAi phenotypes in $r r f-3$ and lin-35 mutants. However, for 244 candidate genes, we reproducibly observed a stronger feeding RNAi phenotype in lin-35(n2239) than 
in $r r f-3$ (pk1426) and WT animals (Supplementary Table $1 \mathrm{~A})$. The majority of genes $(50 / 69)$ for which no RNAi phenotype had been previously reported belonged to this group (according to wormbase version WS153 [66], see Additional file 1, Supplementary Table 1A).

To further validate the specificity of the interactions with lin-35 Rb, we tested the 244 candidate genes in a more extensive panel of RNAi hypersensitive strains, including rrf-3(pk1426), eri-1(mg366), the synMuv B mutants $h p l-$ 2(ok917), lin-15B(n744), lin-35(n2339) as well as lin$37(n 758)$, and double mutant eri-1(mg366); lin$15 B(n 744)$ animals. In agreement with Cui et al [28] but not Wang et al [26], we observed that lin-37(n758) synMuv B animals are RNAi hypersensitive (Table 1). The strain eri-1; lin-15B was included as an extreme RNAi hypersensitive control, with the caveat that this strain is somewhat sick and was found to display a variety of defects with low penetrance (data not shown).

In the combined screens, we identified 57 genes whose RNAi phenotype was reproducibly stronger in lin-35, or in multiple synMuv B mutants, than in WT, rrf-3 and eri-1. These 57 genes can be classified in three groups: 5 genes showed an RNAi phenotype substantially stronger in lin35(n2239) than in any other background, 31 genes displayed strong RNAi phenotypes in lin-35(n2239) and in one or two other synMuv B mutants, and 21 genes showed strong RNAi phenotypes in all synMuv B mutants examined (Figure 1) (see Additional file 1, Supplementary Table 1B). We consider all three classes of interest, as synthetic interactions could either be specific for lin-35 or take place with multiple Rb-pathway components. The group of 31 candidates includes pha-1, which was previously shown to interact with lin-35 [14] and provides a first validation that our approach identified specific interactions. In addition, the various classes of genes identified are in agreement with previous genetic studies that revealed redundant functions of lin-35 in various developmental processes [9-21]. A high percentage of the genes identified are predicted to fulfill roles in transcription, DNA replication and chromatin structure, as was expected for genes that act redundantly with lin-35 Rb (Figure 1CD). Genes involved in protein degradation were also expected to interact with the lin-35 transcriptional repressor. Such genes, as well as genes involved in signaling, cell cycle and RNA metabolism, were all well represented in our screens as in previous screens (Fig. 1C-D, see Additional file 3 ).

Recently, Lehner et al. described the existence of "hub" genes. This group of genes encodes chromatin regulators that modify diverse signaling pathways and therefore genetically interact with many other genes [29]. As lin-35 is expected to function in chromatin remodeling and interacted with a large number of genes in our screen, we suggest that $\mathrm{Rb}$ is one of such "hub" genes.

Importantly, redundant (synthetic) interactions with lin$35 \mathrm{Rb}$ have often been revealed through partial loss-offunction mutations, while complete loss of function of the same genes caused lethality (e.g.: pha-1 [14], psa-1 [16], him-17 [18] and gon-14 [20]; the class A synMuv genes are a clear exception). Consistent with this notion, we injected dsRNA to achieve strong RNAi phenotypes for a sample of 12 candidate genes (including pha-1, the known lin-35 interactor), and all but $z f p-2$ caused lethality/sterility with or without co-injection of lin-35 dsRNA (see Additional file 1, Supplementary Table 1B). Therefore, synthetic interaction with lin-35 Rb may be detected with partial loss-of-function alleles (or by feeding dsRNA) but not in a setting approaching strong lossof-function or null conditions (as may be achieved by injecting dsRNA). Thus, independent validation of these genetic interactions will require appropriate alleles.

Similarly, a feeding RNAi phenotype in an eri-1 or $r r f-3$ mutant background does not rule out genetic interaction with lin-35 Rb. However, genes that show an apparent feeding RNAi phenotype only in lin-35 mutants, and not even in eri-1; lin-15B RNAi hypersensitive mutants, are the best candidates for a functional interaction with lin-35 Rb. Only five genes showed this level of specificity: $z f p-2$, F25H5.5, rsr-2, Y17G7B.2 and tir-1.

F25H5.5 encodes the putative C. elegans homolog of Claspin, which in vertebrates mediates activation of the Chk1 kinase as part of a DNA replication checkpoint response [30]. As pRb and Claspin both negatively regulate cell-cycle progression, a synthetic lethal interaction is conceivable. It will be attractive to examine whether reducing human Claspin activity specifically increases the killing of Rb-minus tumor cells, if not alone then possibly in response to e.g. gamma-irradiation (experiments in progress).

The predicted Y17G7B. 2 product is similar to the trithorax group protein Ash2, a subunit of a histone H3 (Lys4) methyltransferase complex [31]. Genetic interaction between the Ash2 and pRb family members is plausible, as both form part of protein complexes that regulate gene expression through histone modification, and physical interactions between components of these complexes have been reported [32]. Indeed, others recently observed a genetic interaction between mutant alleles of Y17G7B.2/ ash-2 and lin-35 (G. Soete and HC Korswagen, manuscript in preparation), which validates the identification of this gene in our screen. 
Table I: Novel RNAi hypersensitive strains and gene-dependent hypersensitivity

\begin{tabular}{|c|c|c|c|c|c|c|}
\hline & & N2 & $r r f-3$ (pkl426) & lin-35 (n2239) & lin-37 (n758) & $\mathrm{zfp}-2(\mathrm{tm} 557)$ \\
\hline RNAi & Phenotype & & & & & \\
\hline$g p c-2$ & Emb & $0 \%$ & $14.9 \%$ & $2.6 \%$ & $2.8 \%$ & $0 \%$ \\
\hline mom-2 & Emb & $17.8 \%$ & $40.1 \%$ & $64.1 \%$ & $67.4 \%$ & $69.4 \%$ \\
\hline$h m r-I$ & Lvl & $0 \%$ & Ste, Ooc, Emb & $80.7 \%$ & $77.6 \%$ & $53.1 \%$ \\
\hline cel-I & Lva & $0.5 \%$ & $100 \%$ & $98.6 \%$ & $11 \% *$ & $23.2 \%$ \\
\hline unc-87 & Unc & $3.6 \%$ & $0 \%$ & $94.2 \%$ & $85.3 \%$ & $0 \%$ \\
\hline unc- 15 & Unc & $0 \%$ & $100 \%$ & $96.5 \%$ & $92.6 \%$ & $0 \%$ \\
\hline
\end{tabular}

We tested several RNAi clones, previously used by others to quantitatively examine sensitivity to RNAi, in different genetic backgrounds $[26,62,63]$. To allow comparison with RNAi effects reported by others, we used RNAi clones from the Ahringer Library [64] except for cel-I(Vidal library). Numbers are averages of 3 to 6 experiments and indicate proportion of animals (FI progeny) displaying the corresponding phenotype. In agreement with recent findings $[26,65]$, we also observed that different RNAi hypersensitive mutants showed surprising gene specificity in RNAi enhancement.

*cel-I RNAi in lin-37(n758) produces larval arrest at later stages

The tir-1 gene encodes a protein with a conserved Toll/IL1 resistance (TIR) domain and has been shown to act in a conserved MAP kinase pathway in innate immunity $[33,34]$ as well as lateral signaling between specific neurons [35]. The basis of a genetic interaction between tir-1 and lin-35 Rb is not immediately obvious. However, if confirmed it should be of considerable interest, as TIR-1 contains a single human ortholog, SARM. Like TIR-1, SARM contains a TIR domain, heat-armadillo repeats and two sterile alpha motifs (SAM) [35].

The $z f p-2$ gene encodes a zinc-finger transcription factor with six $\mathrm{C} 2 \mathrm{H} 2$ zinc finger domains and a KRAB A domain, which is associated with transcriptional repression [36] (see Additional file 4). Human pRb has been reported to physically interact with two zinc finger proteins named RIZ1 and RBak. RIZ1 is commonly inactivated in human cancers, and RBak contains a KRAB domain and has homology with $z f p-2$ [37-39]. Finally, rsr-2 encodes a serine $(\mathrm{S})$ and arginine (R)-rich protein with homology to the human splicing co-activator Srm300. The $z f p-2$ and $r s r-$ 2 genes and their interactions with lin-35 are discussed below.

\section{The zinc finger zfp-2 cooperates with lin-35 in somatic gonad development}

Of the five candidates that interacted specifically with lin35 (Figure $1 \mathrm{~A}$, top), viable mutations were only available for $z f p-2$ and tir-1. We did not observe a synthetic interaction between lin-35 and the tir-1 deletion alleles ok1052 and $t m 1111$. However, these alleles likely do not cause tir1 inactivation, as the deletions affect specific splice forms while two of the confirmed tir-1 mRNAs (F13B10.1b and d) [66] should be expressed normally. In contrast, we observed a strong synthetic interaction between the $z f p-2$ allele tm557 and the lin-35 candidate null mutations $n 745$ and $n 2239$ as well as lin-35(RNAi). The $z f p-2(t m 557)$ mutation is a $233 \mathrm{bp}$ deletion which removes $130 \mathrm{bp}$ of coding sequences and changes the reading frame shortly after the predicted translation-initiation codon.

Inactivation of $z f p-2$ alone by RNAi (feeding or injection) or the $z f p-2(t m 557)$ homozygous deletion did not cause any apparent abnormalities (Figure 2A,C). Although lin35 mutants are also viable, their brood size is reduced (Figure 2A and [10]). We observed a variety of defects in lin-35(n2239) mutants with low penetrance, including reduced integrity of the proximal gonad (uterus and spermatheca), a low percentage of endomitotic oocytes (Emo), DTC migration defects $(8.4 \%, \mathrm{n}=107)$ and abnormalities in vulval morphology $(3.7 \%$ everted vulva, $\mathrm{n}=$ 107). Loss-of-lin-35 function also allowed continued nuclear division of intestinal nuclei during larval development (variable and partly dependent on developmental stage and temperature, ranging between normal (32-34 nuclei) and 55 nuclei/animal $[[17,19]$, and our unpublished observations].

Importantly, lin-35(n2239); zfp-2(tm557) double mutants were almost completely sterile (Figure 2A). In addition, defects in gonad migration $(18 \% \mathrm{n}=188)$ and vulval morphology $(17 \% \mathrm{n}=188)$ were enhanced compared to lin-35 alone and polyploid nuclei were seen in the soma (Figure $2 \mathrm{H}$ ). Such sterile double mutant animals showed dramatic morphological defects in the proximal somatic gonad, with absence of a normal uterine cavity and, based on misplaced sperm precursors and spermatids, dysfunctional spermatheca (Figure 2D,2F and 2H). Oocytes were present but in general did not ovulate, were not fertilized and often became polyploid (endomitotic oocytes: Emo phenotype [40]) (Figure 2H). These defects resemble phenotypes observed after ablation of somatic cell precursors 


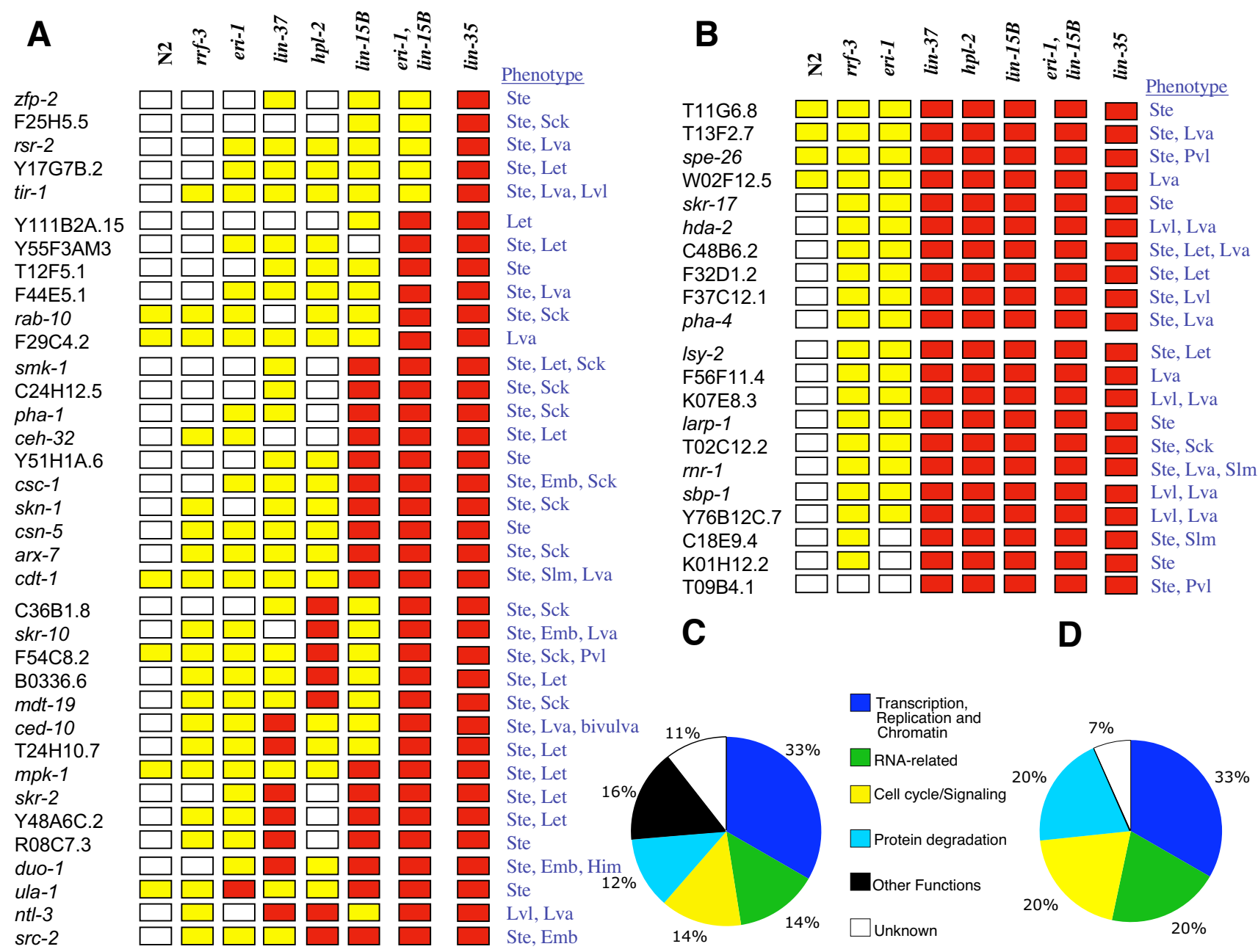

Figure I

(A, B) Color code representation of the strength of RNAi phenotypes of candidate genes in different genetic backgrounds. Red indicates a highly penetrant lethal phenotype. Yellow designates a substantially weaker phenotype and/or increase in viable progeny and white indicates weak or no detectable defects. (A) Synthetic lethal or enhanced RNAi phenotypes. (B) RNAi phenotypes enhanced in synMuv B mutants See: Additional file I, Supplementary Table I, for details. (C) Pie Chart representing the major functional categories among the 57 candidates genes (See Additional file I, Supplementary Table IC). (D) Pie Chart representing major functional categories among 14 genetic interactors previously published (See Additional file 3, Supplementary Figure I).

in the germ line, which indicated that sheath and spermathecal cells are required for ovulation and fertilization [41].

Examination of the $z f p$-2 expression pattern suggested that the affected tissues express both lin-35 and $z f p-2$. While lin-35 Rb seems to be ubiquitously expressed [9,42], zfp-2 expression is more restricted. Transgenic animals expressing the Green Fluorescent Protein (GFP) under the control of the $z f p-2$ promoter showed fluorescence in vulval cells and all somatic gonad structures such as spermatheca, sheath cells, uterine cells and distal tip cells (DTCs) (Figure 3). This expression pattern correlates well with the observed synthetic phenotype. Together, these observations indicate that lin-35 and $z f p-2$ function redundantly in development of the somatic gonad lineages derived from Z1 and Z4 (Figure 2B: DTCs, spermatheca, sheath cells and uterine cells)[43]. This may explain the abnormal migration of DTCs, morphological defects of the uterus and spermatheca, and synthetic sterile phenotype of lin-35(n2239); zfp-2(tm557) double mutants.

Although gonadal defects were apparent, the developmental program is partially completed and cell specification, based on reporter gene expression and structure, does occur (Figure 2F). Some of the sheath cells were 

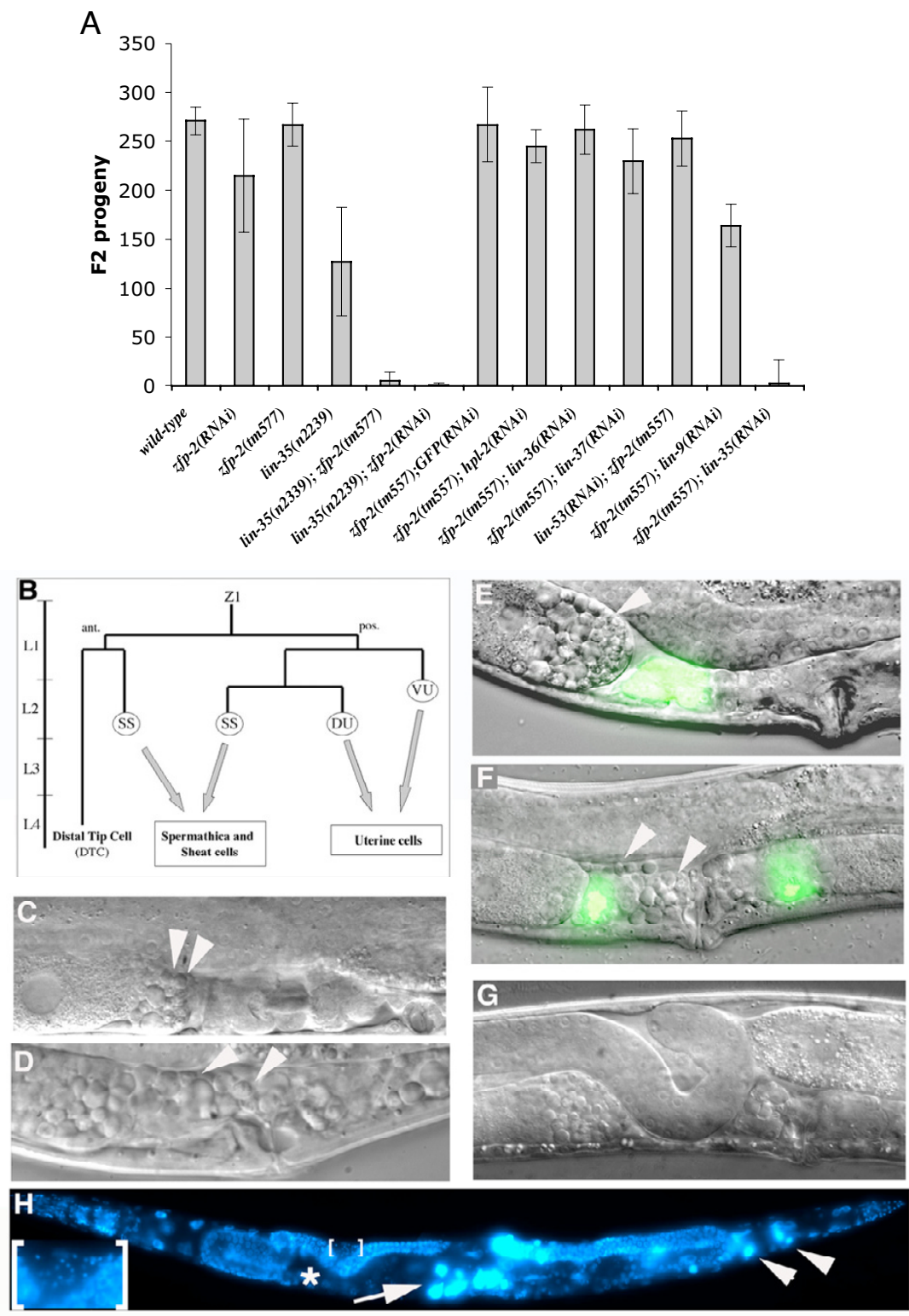

Figure 2

Genetic interaction between zfp-2 and lin-35 Rb. (A) Double inactivation of $z f p-2$ and lin-35 causes sterility. Total numbers of progeny were counted for 77 single lin-35(n2239); zfp-2(tm557) hermaphrodites. For other genotypes, total progeny of 6 to 12 single hermaphrodites were counted. Error bars indicate standard deviation.(B-H) Somatic gonad defects in animals deficient for zfp-2 and lin-35. (B) Simplified scheme representing the lineage of ZI through larval stages, which it is one of the two precursors of the somatic gonad. (ant: anterior, post: posterior, SS: sheath/spermathecal precursor, DU and VU: dorsal and ventral uterine precursors). (C) Detail of WT proximal hermaphrodite gonad. White arrowheads point to spermatocytes and spermatids in panels C-F. (D) Detail of sterile lin-35(n2239); zfp-2(tm557) adult hermaphrodite with defective spermatheca and uterus. Some sperm precursors, spermatocytes and spermatids, are misplaced. (E) Expression of $f k h-6::$ GFP in lin-35 (RNAi) animals. Sperm precursors are properly located. (F) Expression of $k h-6:: G F P$ in lin-35(RNAi); zfp-2(tm557) animals. Sperm precursors are misplaced. Expression of $f k h-6$ is required for normal differentiation of the spermatheca and uterus [60] and appears normal, indicating that cell specification is initiated. (G) Defect in Distal Tip Cell migration in lin-35(n2239); zfp2(tm557) animal. (H) lin-35(n2239); zfp-2(tm557) adult, DNA stained with DAPI. Note aberrant turn of one gonad arm (*), endomitotic oocytes (Emo) (arrow) and polyploid somatic nuclei (arrowheads). Proper proximal gonad structures such as spermatheca are missing. Lower left: High magnification of the bracketed area. Note misplaced sperm (small and intense blue spots). 

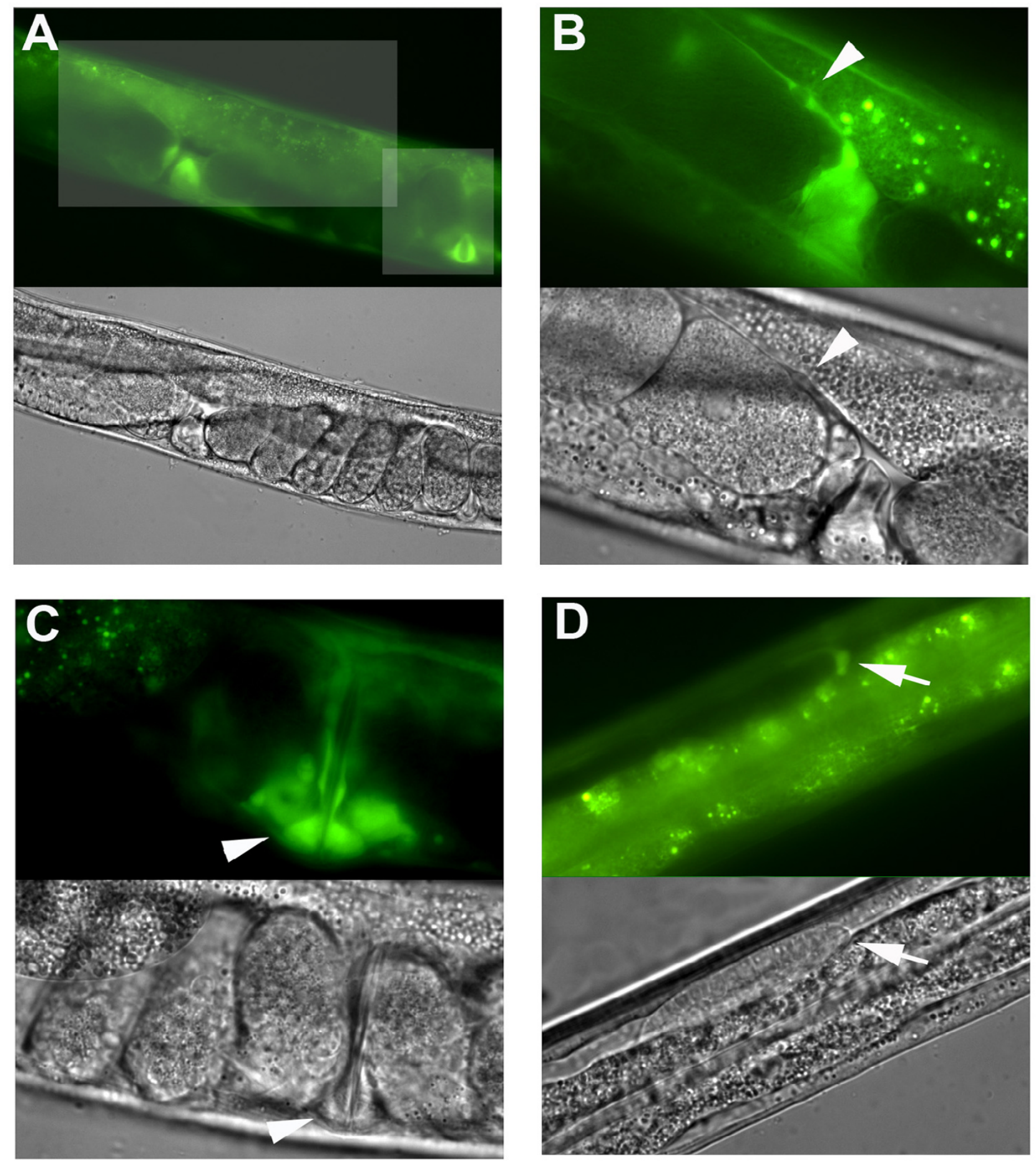

\section{Figure 3}

zfp-2::GFP is expressed in the somatic gonad. (A) zfp-2::GFP expression in somatic gonad tissue and vulval cells. (B) Magnification of the rectangular area highlighted in A. GFP expression in spermatheca and sheath cells. Arrowhead indicates sheath cell. (C) Magnification of the square highlighted in A. GFP expression in several vulval cells. Arrowheads indicate equivalent vulval cells (D) GFP expression in distal tip cell (DTC) (arrow). In addition, we observed GFP expression in some neurons, pharyngeal cells, intestine and tail (not shown). The corresponding light microscopy images are shown below epifluorescence figures. 
apparently missing in the double mutants, but quantitative analysis was hampered by silencing of the sheath cell marker (lim-7::GFP [44]) upon injection of lin-35 and $z f p$ 2 dsRNA (Supplementary Figure 3). Knock down of lin-35 and $z f p-2$ after completion of somatic gonad development did not cause sterility. Specifically, when lin-35 and $z f p-2$ dsRNA were injected in the gonad of L4 larvae or young adult animals, the injected animals remained fertile while their progeny were sterile. Although such experiments do not provide strong evidence, the results are consistent with redundant functions for $l i n-35$ and $z f p-2$ in somatic gonad development, rather than oocyte maturation.

F35H8.3/zfp-2 was previously found in an RNAi screen as one of 59 genes required for cosuppression [45]. Cosuppression refers to the coincident silencing of a repetitive transgene and corresponding endogenous gene, which involves chromatin remodeling and RNAi pathway components [45]. Remarkably, we observed that $z f p-2$ (tm557) mutants are not RNAi resistant but hypersensitive to RNAi for certain genes (Table 1). Thus, $z f p-2$ and lin-35 both suppress RNAi, which indicates a more extensive functional relationship. As yet another similarity with lin-35, $z f p-2$ RNAi also caused silencing of the rol-6 transgene (see Additional file 5). Based on the similarities and genetic interaction with lin-35, we favor the model that ZFP-2 acts as a zinc-finger transcription factor in transcriptional suppression.

In conclusion, $z f p$-2 and lin-35 show a synthetic lethal interaction and cooperate in development of the somatic gonad, likely acting in parallel or together in transcription repression and/or chromatin remodeling.

\section{Knock down of specific splicing components resembles the lin-35 $\boldsymbol{R b}$ phenotype}

The synMuv B gene lin-35 $\mathrm{Rb}$ represses ectopic induction of the vulval cell fate redundantly with synMuv A and $C$ genes. We did not observe synthetic Multivulva phenotypes in our screen, not even for the two known class A genes or single class $\mathrm{C}$ gene present in the library. This fits with our observations that class A genes tend to score false negative in feeding RNAi screens (our unpublished results).

Although synthetic interaction between null alleles often points to functions in parallel pathways, several observations indicate that class A and B synMuv proteins could in fact act in conjunction $[46,47]$. Similarly, genetic interaction with lin-35 Rb in our screen could reveal genes that act in parallel pathways, but possibly also identify additional components of a LIN-35 protein complex or pathway. As such, it was of interest that a known synMuv B gene, mep-1, was found in the set of 244 candidates. To examine whether additional synMuv B genes were present among the putative lin-35 interactors, we performed feeding RNAi for the 244 candidates in the synMuv A mutant lin-15A(n767). This screen identified $r s r-2$, one of the five genes that showed a feeding RNAi phenotype specifically in lin-35 mutants (Figure 1A), as a novel synMuv B gene.

Despite the lin-35 specific feeding RNAi phenotype, $r s$-2 dsRNA injection caused severe abnormalities such as sterility, embryonic lethality and larval arrest [[48], and this report]. Interestingly, rs -2 encodes a serine (S) and arginine (R)-rich protein with homology to the human splicing co-activator Srm300, which acts with other splicing factors to perform its functions. To test whether additional splicing factors belong to the synMuv B family, we collected feeding RNAi clones corresponding to 135 putative C. elegans splicing-related genes (see Additional file 2, Supplementary Table 2A; legend, see Additional file 6). We assayed these 135 genes by feeding RNAi in L1 and young adult lin-15A mutants (see Additional file 2, Supplementary Table $2 \mathrm{~B}$ ). An additional nine genes showed a Muv phenotype in lin-15A and other class A mutants (Table 2), but not in wild-type or class B animals, classifying these genes as synMuv B. These nine genes include the seven members of the Sm family in C. elegans (snr-1, 2, 3, 4, 5, 6 and 7) [49] as well as two Sm-like genes (lsm-2/gut2 and $l s m-4)$, all of which are well conserved through evolution. Only $l s m-2$ and $l s m-4$ RNAi produced viable animals at $25^{\circ} \mathrm{C}$, at which temperature the synMuv phenotype is most highly penetrant. Combination of $l \mathrm{sm}$ 2 or $l s m-4$ RNAi with a mutation in any one of four different Class A genes caused a Muv phenotype, ranging from $20 \%$ to $96 \%$ of the animals at $25^{\circ} \mathrm{C}$ (Table 2 ). Genetic epistasis analysis revealed that this splice factor associated synMuv B phenotype depends on a functional let-60 Ras signaling pathway and is reduced in lin-3 EGF mutants, in agreement with genetic epistasis analysis of other synMuv B genes (Table 2) [9,50].

In addition to their role in vulval-fate determination, synMuv B genes show specific combinations of other loss-offunction characteristics that include: deregulation of cellcycle entry, ectopic expression or silencing of transgenes, RNAi hypersensitivity and expression of germline P granules in the soma [26,51]. Although the ten splicing-related synMuv B genes are essential for viability, partial inactivation by feeding RNAi induced other properties of synMuv $\mathrm{B}$ genes (Table 3). For example, like lin-35 inactivation (see above), $r s r$-2 RNAi led to formation of extra intestinal nuclei $(41.3 \pm 3.6(n=37)$ versus control: $33.3 \pm 1.9(n=$ 27 ) in the elt-2::GFP strain) (Figure 3 ). In addition, RNAi of several synMuv B genes induces ectopic expression of a lag-2::GFP reporter in the intestine [51] and we observed the same phenotype after RNAi of $l s m-2$ and $l s m-4$, a weaker phenotype after RNAi of $s n r$ genes, but no effect after $r$ sr-2 RNAi (Table 3). Moreover, like lin-35 loss of 
Table 2: Novel synMuv B genes and genetic epistasis analysis.

\begin{tabular}{|c|c|c|c|c|c|c|}
\hline & \multicolumn{4}{|c|}{$\%$ of animals with $2,3,4$ or 5 pseudovulvae } & \multirow[b]{2}{*}{$\%$ of Muv } & \multirow[b]{2}{*}{ n } \\
\hline & 2 & 3 & 4 & 5 & & \\
\hline \multicolumn{7}{|c|}{ synMuv $B$ activity } \\
\hline$r s r-2(R N A i)$ & $0 \%$ & $0 \%$ & $0 \%$ & $0 \%$ & $0 \%$ & 445 \\
\hline rsr-2(RNAi); lin-I 5A(n767) & $8.6 \%$ & $1.3 \%$ & $0 \%$ & $0 \%$ & $10 \%$ & 453 \\
\hline$r s r-2(R N A i) ; \operatorname{lin}-8(n I I I)$ & $1.5 \%$ & $0 \%$ & $0 \%$ & $0 \%$ & $2 \%$ & 197 \\
\hline$r s r-2(R N A i) ; \operatorname{lin}-38(n 75 I)$ & $1.0 \%$ & $0.1 \%$ & $0 \%$ & $0 \%$ & $1 \%$ & 787 \\
\hline rsr-2(RNAi); lin-56(n2728) & $2.0 \%$ & $0 \%$ & $0 \%$ & $0 \%$ & $2 \%$ & 595 \\
\hline rrf-3(pk I 426); Ism-2(RNAi) & $0 \%$ & $0 \%$ & $0 \%$ & $0 \%$ & $0 \%$ & $>300$ \\
\hline Ism-2(RNAi); lin-I 5A(n767) & $1.5 \%$ & $3.1 \%$ & $1.0 \%$ & $0 \%$ & $6 \%$ & 390 \\
\hline Ism-2(RNAi) $25^{\circ} \mathrm{C}$ & $0 \%$ & $0 \%$ & $0 \%$ & $0 \%$ & $0 \%$ & 220 \\
\hline Ism-2(RNAi); lin-I $5 \mathrm{~A}(n 767) 25^{\circ} \mathrm{C}$ & $13.8 \%$ & $42.5 \%$ & $37.8 \%$ & $0.5 \%$ & $94 \%$ & 188 \\
\hline Ism-2(RNAi); lin-8(nIII) $25^{\circ} \mathrm{C}$ & $14.7 \%$ & $9.5 \%$ & $1.0 \%$ & $1.0 \%$ & $26 \%$ & 136 \\
\hline Ism-2(RNAi); lin-38(n75I) $25^{\circ} \mathrm{C}$ & $13.4 \%$ & $27.4 \%$ & $17.8 \%$ & $0 \%$ & $60 \%$ & 157 \\
\hline Ism-2(RNAi); lin-56(n2728) $25^{\circ} \mathrm{C}$ & $7.1 \%$ & $8.2 \%$ & $4.7 \%$ & $0 \%$ & $20 \%$ & 85 \\
\hline rrf-3(pk I 426); Ism-4(RNAi) & $0 \%$ & $0 \%$ & $0 \%$ & $0 \%$ & $0 \%$ & $>300$ \\
\hline Ism-4(RNAi); lin-I $5 A(n 767)$ & $2.4 \%$ & $5.0 \%$ & $0.5 \%$ & $0 \%$ & $8 \%$ & 420 \\
\hline Ism-4(RNAi) $25^{\circ} \mathrm{C}$ & $0 \%$ & $0 \%$ & $0 \%$ & $0 \%$ & $0 \%$ & 149 \\
\hline Ism-4(RNAi); lin-I5A(n767) $25^{\circ} \mathrm{C}$ & $16.0 \%$ & $40.8 \%$ & $38.3 \%$ & $0.6 \%$ & $95 \%$ & 162 \\
\hline Ism-4(RNAi); lin-8(nIII) $25^{\circ} \mathrm{C}$ & $11.7 \%$ & $9.0 \%$ & $1.4 \%$ & $0 \%$ & $22 \%$ & 145 \\
\hline Ism-4(RNAi); lin-38(n75I) $25^{\circ} \mathrm{C}$ & $10.5 \%$ & $45.3 \%$ & $23.2 \%$ & $0 \%$ & $79 \%$ & 95 \\
\hline Ism-4(RNAi); lin-56(n2728) $25^{\circ} \mathrm{C}$ & $13.2 \%$ & $14.0 \%$ & $5.3 \%$ & $0 \%$ & $33 \%$ & 114 \\
\hline lin-37(RNAi); lin-I 5A(n767) & $27.6 \%$ & $41.4 \%$ & $24.1 \%$ & $6.9 \%$ & $100 \%$ & 29 \\
\hline lin-35(RNAi); lin-I 5A(n767) & $0 \%$ & $27.5 \%$ & $62.5 \%$ & $10.0 \%$ & $100 \%$ & 40 \\
\hline lin-35(RNAi); lin-38(n75I) & $1.6 \%$ & $1.6 \%$ & $67.2 \%$ & $22.9 \%$ & $93 \%$ & 61 \\
\hline $\operatorname{lin}-35(R N A i) ; \operatorname{lin}-15 A(n 767) 25^{\circ} \mathrm{C}$ & $0 \%$ & $0 \%$ & $33.7 \%$ & $58.7 \%$ & $92 \%$ & 92 \\
\hline $\operatorname{lin}-35(R N A i) ; \operatorname{lin}-8(n I I I) 25^{\circ} \mathrm{C}$ & $1.2 \%$ & $7.1 \%$ & $29.4 \%$ & $0 \%$ & $37 \%$ & 85 \\
\hline lin-35(RNAi); lin-56(n2728) $25^{\circ} \mathrm{C}$ & $1.2 \%$ & $6.1 \%$ & $37.8 \%$ & $45.1 \%$ & $89 \%$ & 82 \\
\hline snr-I (RNAi)(LI)(MV) & $0 \%$ & $0 \%$ & $0 \%$ & $0 \%$ & $0 \%$ & $>200$ \\
\hline
\end{tabular}


Table 2: Novel synMuv B genes and genetic epistasis analysis. (Continued)

\begin{tabular}{|c|c|c|c|c|c|c|}
\hline lin-35(n2239); snr-I (RNAi)(LI)(MV) & $0 \%$ & $0 \%$ & $0 \%$ & $0 \%$ & $0 \%$ & $>200$ \\
\hline snr-I(RNAi)(LI)(MV); lin-I 5A(n767) & $9.3 \%$ & $3.6 \%$ & $4.3 \%$ & $0.4 \%$ & $18 \%$ & 278 \\
\hline snr-I (RNAi)(LI)(JA); lin-I 5A(n767) & $1.6 \%$ & $3.2 \%$ & $0 \%$ & $0 \%$ & $5 \%$ & 62 \\
\hline snr-I (RNAi)(LI)(MV); lin-56(n2728) & $2.4 \%$ & $1.4 \%$ & $0.7 \%$ & $0 \%$ & $5 \%$ & 293 \\
\hline snr-I (RNAi)(LI)(MV); lin-38(n75I) & $3.3 \%$ & $1.1 \%$ & $0 \%$ & $0 \%$ & $4 \%$ & 132 \\
\hline snr-2(RNAi)(LI)(MV); lin-I 5A(n767) & $1.0 \%$ & $1.0 \%$ & $0 \%$ & $0 \%$ & $2 \%$ & 93 \\
\hline snr-3(RNAi)(LI)(MV) & $0 \%$ & $0 \%$ & $0 \%$ & $0 \%$ & $0 \%$ & $>200$ \\
\hline lin-35(n2239); snr-3(RNAi)(LI)(MV) & $0 \%$ & $0 \%$ & $0 \%$ & $0 \%$ & $0 \%$ & $>200$ \\
\hline snr-3(RNAi)(LI)(MV); lin-I 5A(n767) & $14.0 \%$ & $4.0 \%$ & $0.5 \%$ & $0 \%$ & $19 \%$ & 221 \\
\hline snr-3(RNAi)(LI)(MV); lin-38(n75I) & $2.4 \%$ & $0.8 \%$ & $0 \%$ & $0 \%$ & $3 \%$ & 126 \\
\hline snr-4(RNAi)(LI)(MV); lin-I 5A(n767) & $9.7 \%$ & $4.8 \%$ & $1.2 \%$ & $0 \%$ & $16 \%$ & 83 \\
\hline snr-4(RNAi)(LI)(JA); lin-I 5A(n767) & $7.1 \%$ & $3.2 \%$ & $0 \%$ & $0 \%$ & $10 \%$ & 156 \\
\hline snr-5(RNAi)(LI)(MV); lin-I 5A(n767) & $1.3 \%$ & $1.3 \%$ & $0.6 \%$ & $0 \%$ & $3 \%$ & 158 \\
\hline snr-6(RNAi)(LI)(MV); lin-I 5A(n767) & $4.7 \%$ & $3.5 \%$ & $0 \%$ & $0 \%$ & $8 \%$ & 257 \\
\hline snr-7(RNAi)(LI)(MV); lin-I 5A(n767) & $10.7 \%$ & $7.1 \%$ & $2.2 \%$ & $0 \%$ & $20 \%$ & 140 \\
\hline
\end{tabular}

\section{EGF/RAS/MAPK epistasis}

rsr-2(RNAi); let-23(sy97); lin-I5A(n767)

$\begin{array}{llllll}0 \% & 0 \% & 0 \% & 0 \% & 0 \% & 99\end{array}$

rsr-2(RNAi); let-60(n /876); lin-I 5A(n767)

$\begin{array}{llllll}0 \% & 0 \% & 0 \% & 0 \% & 0 \% & 43\end{array}$

rsr-2(RNAi); lin-3(n378); lin-I 5A(n767)

$5.0 \% \quad 1.3 \% \quad 0 \%$

$0 \% \quad 0 \%$

$6 \%$

80

let-60(n/876); Ism-2(RNAi); lin-I 5A(n767)

lin-3(n378); Ism-2(RNAi); lin-I 5A(n767)

$0 \% \quad 0 \% \quad 0 \%$

$0 \% \quad 0 \%$

$0 \%$

34

$\begin{array}{llllll}1.6 \% & 0 \% & 0 \% & 0 \% & 2 \% & 250\end{array}$

Ism-4(RNAi); let-60(n/876); lin-I5A(n767)

$0 \% \quad 0 \% \quad 0 \%$

$0 \% \quad 0 \%$

$0 \% \quad 0 \%$

64

Ism-4(RNAi); lin-3(n378); lin-I 5A(n767)

$2.7 \%$

$0.6 \% \quad 0 \%$

$0 \%$

$3 \%$

180

lin-35(RNAi); let-23(sy97); lin-I 5A(n767)

lin-35(RNAi); let-60(n/876); lin-I5A(n767)

lin-35(RNAi); lin-3(n378); lin-I5A(n767)

$\begin{array}{cccccc}0 \% & 0 \% & 0 \% & 0 \% & 0 \% & 58 \\ 0 \% & 0 \% & 0 \% & 0 \% & 0 \% & 25 \\ 3.2 \% & 15.9 \% & 74.6 \% & 6.3 \% & 100 \% & 63\end{array}$

RNAi experiments were performed at $20^{\circ} \mathrm{C}$ on young adults unless indicated otherwise. Multivulva animals were scored in the next generation after 5,7 and 9 days. All synMuv A mutants were $0 \%$ Muv at $20^{\circ} \mathrm{C}$ and $25^{\circ} \mathrm{C}$. (LI): feeding RNAi started at first larval stage. (JA): clone from RNAi library generated by the Ahringer lab. (MV): clone from RNAi library generated by the Vidal lab. 
Table 3: Summary of RNAi phenotypes of splicing related synMuv genes in synMuv B activity, transgene silencing (scm::GFP), ectopic expression of lag-2::GFP reporter, extra intestinal cells (elt-2::GFP), ectopic $P$ granules, and rescue of cyd- $I$.

\begin{tabular}{|c|c|c|c|c|c|}
\hline & lin-35 & $r s r-2$ & Ism-2 & Ism-4 & snr's (I-7) \\
\hline RNAi phenotype in WT & Rbs & Ste, Lva & WT & WT & Emb, Lva, Lvl \\
\hline synMuv B & +++ & + & + & + & + \\
\hline synMuv B $\left(25^{\circ} \mathrm{C}\right)$ & +++ & $\mathrm{n} / \mathrm{a}$ & ++ & ++ & $\mathrm{n} / \mathrm{a}$ \\
\hline Transgene silencing $\left(25^{\circ} \mathrm{C}\right)$ & ++ & - & - & - & $\mathrm{n} / \mathrm{a}$ \\
\hline Ectopic lag-2::GFP & + & - & - & - & + \\
\hline Ectopic lag-2::GFP $\left(25^{\circ} \mathrm{C}\right)$ & ++ & - & ++ & ++ & + \\
\hline Extra intestinal cells & + & + & - & - & - \\
\hline Ectopic P granules & +++ & - & - & - & + \\
\hline cyd-I rescue & + & - & - & - & - \\
\hline
\end{tabular}

Summary of RNAi phenotypes of splicing related genes: synMuv B activity, transgene silencing (scm::GFP), ectopic expression of lag-2::GFP reporter, extra intestinal cells (elt-2::GFP), ectopic $\mathrm{P}$ granules, and rescue of cyd-I. If $25^{\circ} \mathrm{C}$ is not indicated, experiments were performed at $20^{\circ} \mathrm{C}$. "+++", "++", "+" and "-"stand for strong, moderate, low and no effect respectively. (n/a: not annotated).

function, feeding RNAi of snr-2 and snr-3 induced ectopic P-granules in the soma (Figure 4). lin-35 RNAi also causes silencing of the scm::GFP transgene and partial rescue of cyd-1 larval arrest. These latter effects were not observed after RNAi of the ten novel synMuv B genes. Together, the observed effects suggest that components of the general splicing machinery contribute surprisingly specific functions to some, but not all, processes mediated by lin-35 Rb and other synMuv B genes (Table 3).

How can spliceosome components behave as synMuv B genes? Evidently, complete inactivation of splicing should cause loss-of-function of all intron-containing genes, including synMuv B genes. However, we used partial lossof-function conditions that should not completely block splicing, and three of the phenotypic readouts involve increased, rather than reduced, expression levels. Specifically, the synMuv phenotype likely depends on increased expression of lin-3 EGF in the hyp7 hypodermal syncytium [50], extra nuclear divisions require induction of $S$ phase genes and ectopic lag-2 expression needs de novo expression of the transgene reporter. Importantly, RNAi for many other critical splicing components did not cause a Multivulva phenotype (see Additional file 2, Supplementary Table $2 \mathrm{~B}$ ), indicating that this phenotype is not a consequence of a general shutdown of the splicing process. A more specific defect in splicing of synMuv B premRNAs cannot be excluded. However, it is unclear why splicing of one or more synMuv B messages would depend strongly on a subset of general splicing factors, and not on many others. Moreover, because the splicing related genes display non-overlapping synMuv B features, a single synMuv B pre-mRNA that is particularly sensitive to (alternative) splicing does not provide an explanation for the observed phenotypes (Table 3).

Functions independent from splicing have been reported for Sm and Sm-like (LSM) proteins and should be considered as alternative explanations [49,52-54]. Studies in C. elegans have shown that Sm proteins are components of germline specific $P$ granules and regulate multiple aspects of the germ-cell fate $[49,52]$. While LSM proteins were not examined, these studies indicated that Sm ribonucleoprotein complexes can act distinct from pre-mRNA splicing, possibly in the posttranscriptional control of maternal mRNAs. Recently, cytoplasmic structures known as P bodies or GW bodies have been linked to mRNA degradation and storage [53]. Yeast and human LSM 1-7 proteins have been shown to localize to these cytoplasmic bodies and have been implicated in the degradation of mRNA [54]. Thus, the synMuv B activity of specific splicing related proteins could relate to alternative functions in mRNA metabolism and translation. Such functions could inhibit gene expression post-transcriptionally, in concert with transcriptional repression mediated by LIN-35 Rb complexes. If this model is correct, it could indicate a surprising overlap between repression of gene expression at the transcriptional level in the nucleus and post-transcriptional level in the cytoplasm.

Of great interest, a link between mRNA processing bodies and the microRNA (miRNA) and RNAi pathways has recently been established [55-57]. In addition, RNA-interference related pathways have been observed to cooperate with lin-35 Rb [19], and Sm proteins have also been found to interact with the RNAi machinery in C. elegans $[58,59]$. Specifically: while snr-7 is required for RNAi and transgene expression [58], SNR-3 physically interacts with DCR-1, a key enzyme in the RNAi and miRNA pathways [59]. Like lin-35 and $r s r$-2 (this study), dcr-1 also negatively regulates nuclear divisions in the intestine [19]. Thus, cooperation between LIN-35 Rb and Sm/LSm protein functions might involve miRNA/siRNA pathways.

Further studies will be needed to examine potential coupling between transcription, splicing and RNAi-related processes. Based on the overlapping loss-of-function phenotypes, we propose a model in which specific splicing 

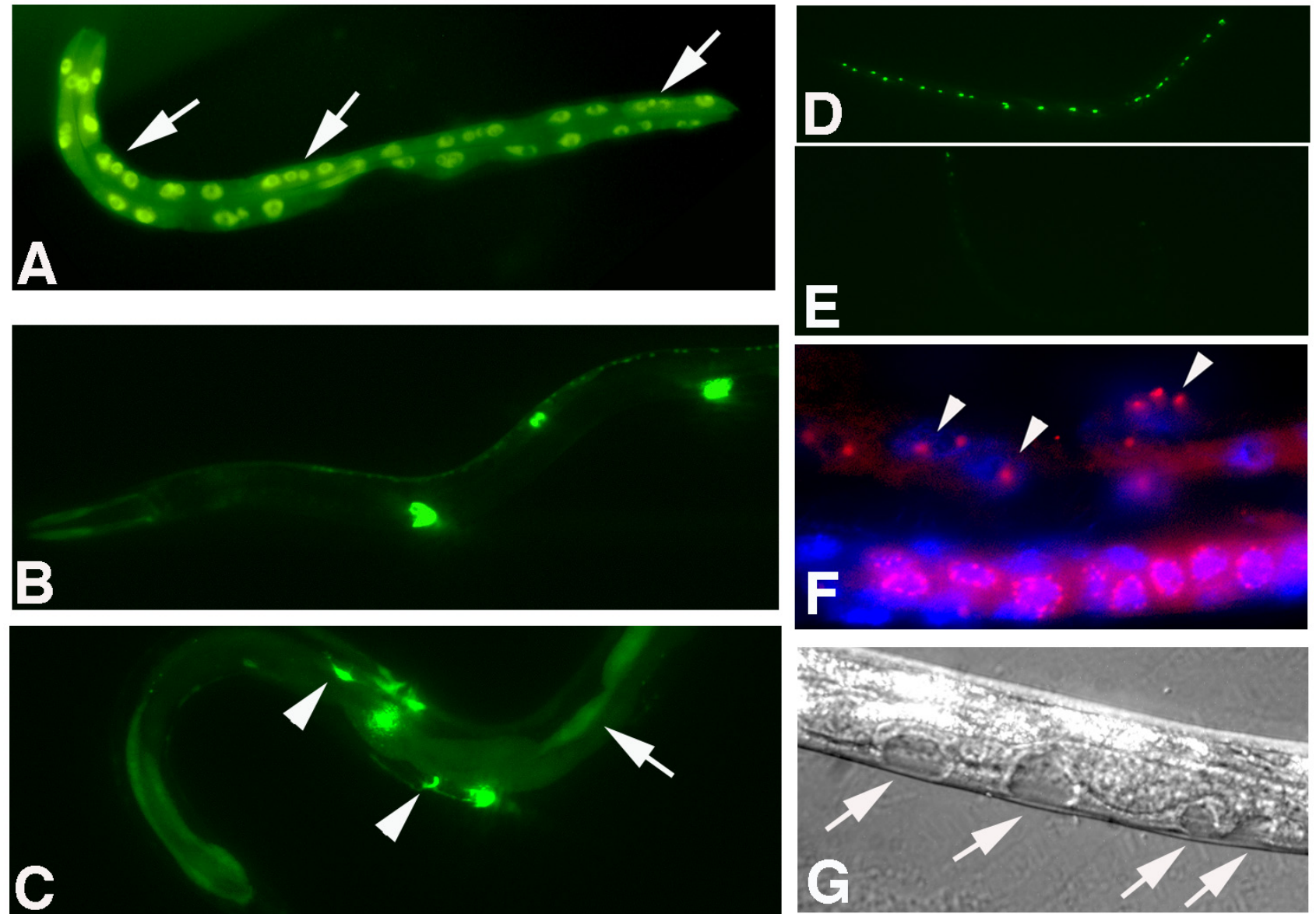

Figure 4

Inactivation of specific spliceosome components resembles synMuv B genes. (A) rsr-2 RNAi by feeding in WT (N2) animals results in extra intestinal nuclei (arrows) (this animal: 4I nuclei), as visualized by intestine specific expression of elt2::GFP (B) lag-2::GFP reporter shows expression in distal tip cells and vulval cells (C) Ectopic expression of lag-2::GFP reporter in the intestine (arrows) and other cells (arrowheads) after Ism-2 RNAi. (D) Expression of scm::GFP in seam cells is not affected by Ism-4 RNAi. (E) lin-35 RNAi induced silencing of the scm::GFP transgene. (F) snr-3 RNAi by feeding in WT animal induced ectopic $P$ granules in somatic cells (arrowheads), in addition to germ cell precursor (bottom). DAPI staining of DNA is in blue, staining of $P$ granules detected with the K76 antibody in red. (G) Induction of ectopic vulval structures following snr-4 RNAi in lin-I 5 A mutant larvae (L3 stage).

components cooperate with lin-35 synMuv B complexes in repression of gene expression, either by promoting mRNA turnover/translation inhibition or by taking part in multi-protein complexes that regulate transcription, chromatin remodeling, and/or miRNA/RNAi mediated posttranscriptional gene silencing (Figure 5).

\section{Conclusion}

Our results provide an extensive series of candidate lin-35 interacting genes, validate $z f p-2$ as a novel lin-35 synthetic lethal gene, and implicate a subset of spliceosome components in gene regulation in conjunction with the lin-35 Rb pathway.

\section{Methods \\ Strains}

We used the wild-type strain Bristol N2 and the following mutations:

LG I: $\operatorname{lin}-35(n 745, n 2239)$. LG II: $r r f-3(p k 1426)$, $z f p-$ 2(tm557), let-23(sy97). LG III: lin-37(n758), hpl-2(ok917). LG IV: eri-1(mg366), let-60(n1876), unc-22(s7), lin3(n378). LG X: lin-15(n744), lin-15A(n767)

Integrated arrays: rtIs14 [elt-2::GFP; osm-10::HT150Q] IV (a gift from P. W. Faber and A. Hart). ezIs2 [fkh-6::GFP + unc-119(+)] [39]. In addition, we used the integrated array ccIs4251 [myo-3::Ngfp-lacZ, pSAK4(myo-3::Mtgfp)], 


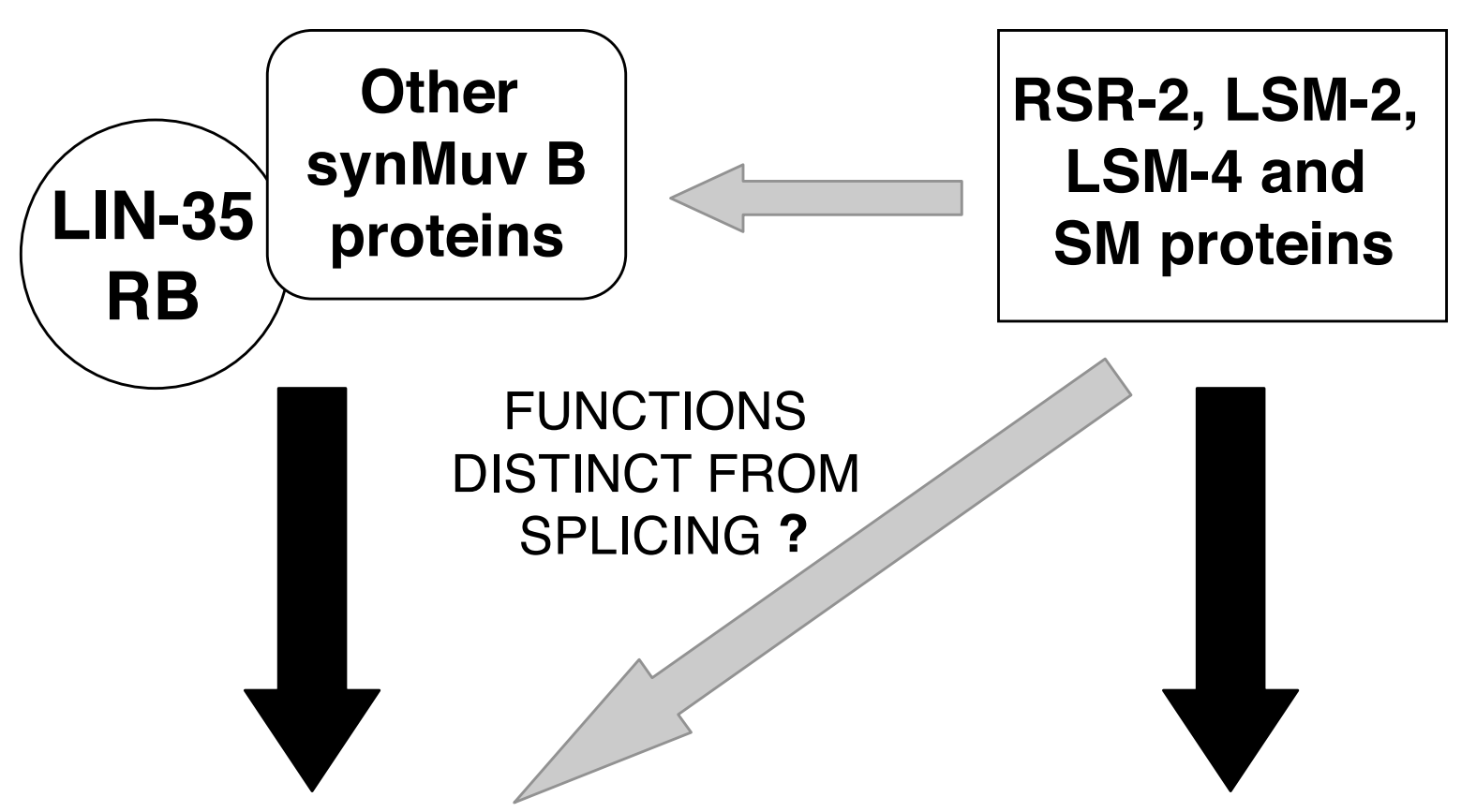

\section{Chromatin remodeling Transcriptional regulation miRNA/RNAi pathways mRNA levels}

\section{Figure 5}

Novel roles of specific spliceosome components in the synMuv B pathway. RSR-2, LSM-2, LSM-4 and SM proteins may have functions that reduce gene expression and are independent of pre-mRNA splicing. The phenotypic overlap with lin-35 $\mathrm{Rb}$ mutants may indicate functions in transcription repression, chromatin modification, miRNA/RNAi pathway modulation or mRNA metabolism.

mapped to LGI (+2.5), to balance lin-35 (n2239) in strain lin-35(n2239)/ccIs4251 I, zfp-2(tm557) II.

\section{RNAi screens}

RNAi experiments were performed as detailed in Rual et al. [25]. In the screen, dsRNA was delivered by feeding L1 synchronized animals on agar plates containing IPTG (6 $\mathrm{mM}$ ) plus ampicillin and tetracycline. 10,953 RNAi clones were tested in 24-well plates. In all RNAi experiments, wild-type animals and lin-35 mutants were simultaneously examined in duplicate (thus, 6 different genes were tested per 24-well plate). RNAi phenotypes were scored visually on days 4 and 6 after L1 worms were put to culture. Because of a substantial variation in feeding RNAi phenotypes, the large number of genes tested and range of phenotypes scored, quantitative determination of phenotypes was unpractical. Therefore, candidates were selected based on obvious and reproducibly stronger phenotypes in lin-35 mutants, as compared to other backgrounds described in the text. For all genes of interest, inserts of feeding RNAi vectors were checked by PCR amplification and DNA sequence analysis.

For many genes, a low penetrant RNAi phenotype was observed that became much stronger when combined with inactivation of lin-35 Rb. We refer to such phenotypes as "enhanced", rather than "synthetic", in combination with lin-35. Lethal (Let) is defined as animals unable to produce viable progeny. When possible, a more informative description is used: Emb (embryonic lethal), Lvl (larval lethal), Lva (larval arrest), or Ste (sterile). Other phenotypes scored were: Unc (uncoordinated), Prl (paralyzed), Dpy (dumpy), Bmd (body morphology defective), Sck (sick), Bli (blistered), Mlt (molting defective), Slm 
(slim), Him (high incidence of males), Pvl (protruding vulva), Muv (multivulva), Clr (clear), Slu (sluggish), Lon (long), Sma (small), Gro (growth rate abnormal), Egl (egg laying defective), Rbs (reduced brood size), Ooc (oocyte formation abnormal), Stp (sterile progeny), and Rup (ruptured). See Additional file 1, Supplementary Table 1, for further information and details.

To screen for synMuv B genes among splicing-related genes, we performed feeding RNAi starting from L4 and L1 stage animals.

\section{dsRNA injection}

Preparation of dsRNA for injection: DNA templates were amplified by PCR from L4440 plasmids using the following primers:

\section{pL440-dest-RNAi-FOR}

(5'GTTTTCCCAGTCACGACGTT3') and pL440-destRNAi-REV (5'TGGATAACCGTATTACCGCC3'). PCR products were used as the templates for synthesis of dsRNA with an in vitro transcription kit (T3 T7 Ambion). The RNA were incubated at $72{ }^{\circ} \mathrm{C}$ for 10 minutes followed by 30 minutes at $37^{\circ} \mathrm{C}$ to allow annealing. The quality and amount of dsRNA was examined by electrophoresis. For injection, we used dsRNA at a concentration of 0.5 to $2 \mu \mathrm{g} / \mu \mathrm{l}$.

\section{Immunostaining}

Worm larvae were permeabilized on coverslips using freeze/cracking. Samples were fixed in ice-cold methanol (10 min.) followed by ice-cold acetone (10 min.). K76 mouse monoclonal antibodies were used $(1: 2)$ to detect $P$ granules. Rhodamine-conjugated anti-mouse antibodies were used as secondary antibodies (1:200).

For staining of DNA, animals were fixed in Carnoys fixative at $4^{\circ} \mathrm{C}$ for 24 hours. After rehydration and washes, samples were incubated for 10 minutes in a solution of 1 $\mu \mathrm{g} / \mathrm{ml}$ DAPI.

\section{Analysis of zfp-2 expression}

$z f p-2::$ GFP transcriptional fusions were generated by in vitrorecombination using the promoterome clones and the destination vector pdest-DD03 as described in Dupuy et al. [61]. Clone and primers sequences can be retrieved from Promoterome database [67].

\section{Authors' contributions}

JC conducted most of the experiments. JFR and MV generated and provided the RNAi library. AC participated in some of the RNAi screens. DD generated the $z f p-2$ expression vector. SV conceived the study, and participated in its design and coordination. JC and SV wrote the manuscript. All authors have read and approved the final manuscript.

\section{Additional material}

\section{Additional File 1}

Supplementary Table 1. Results from RNAi screens and functional categories of candidates genes. (additional legend: Additional file 6) Click here for file

[http://www.biomedcentral.com/content/supplementary/1471213X-7-30-S1.xls]

\section{Additional File 3}

Figure S1. 14 genes previously shown to interact with lin-35. Click here for file [http://www.biomedcentral.com/content/supplementary/1471213X-7-30-S3.pdf]

\section{Additional File 4}

Figure S2. zfp-2 gene structure and KRAB domain conserved sequence. Click here for file

[http://www.biomedcentral.com/content/supplementary/1471213X-7-30-S4.pdf]

\section{Additional File 5}

Figure S3. zfp-2, as lin-35, is required for expression of extrachromosomal arrays.

Click here for file

[http://www.biomedcentral.com/content/supplementary/1471213X-7-30-S5.pdf]

\section{Additional File 2}

Supplementary Table 2. Splicing related genes and associated RNAi phenotypes.

Click here for file

[http://www.biomedcentral.com/content/supplementary/1471-

213X-7-30-S2.xls]

\section{Additional File 6}

Additional legends. Additional legends for additional files 1 and 2. Click here for file

[http://www.biomedcentral.com/content/supplementary/1471213X-7-30-S6.pdf]

\section{Acknowledgements}

We thank Mike Boxem and Erick Morris for critical reading of the manuscript, Kursheed Wani for technical assistance, John Satterlee and members of the Hart and van den Heuvel laboratories for helpful discussions. We are grateful to the Hart, Ruvkun and Horvitz laboratories, the Caenorhabditis Genetics Center and the National Bioresource Project for $C$. elegans (Japan) for strains and information. This research was supported by "AstraZeneca's Academic Sponsorship Program" and the National Institutes of Health (POI CA9528I). JC was a recipient of a postdoctoral fellowship from the Spanish Government (MEC).

\section{References}

I. Friend SH, Bernards R, Rogelj S, Weinberg RA, Rapaport JM, Albert DM, Dryja TP: A human DNA segment with properties of the gene that predisposes to retinoblastoma and osteosarcoma. Nature 1986, 323(6089):643-646.

2. Weinberg RA: The retinoblastoma protein and cell cycle control. Cell 1995, 8 I(3):323-330.

3. Sherr C]: Cancer cell cycles. Science 1996, 274(5293):1672-1677. 
4. Classon M, Harlow E: The retinoblastoma tumour suppressor in development and cancer. Nat Rev Cancer 2002, 2(I 2):910-917.

5. Dimova DK, Stevaux O, Frolov MV, Dyson NJ: Cell cycle-dependent and cell cycle-independent control of transcription by the Drosophila E2F/RB pathway. Genes Dev 2003, I 7( 18):2308-2320.

6. Frolov MV, Dyson NJ: Molecular mechanisms of E2F-dependent activation and pRB-mediated repression. J Cell Sci 2004, I I 7(Pt | I):2|73-8I.

7. Korenjak M, Taylor-Harding B, Binne UK, Satterlee JS, Stevaux O, Aasland R, White-Cooper H, Dyson N, Brehm A: Native E2F/RBF complexes contain Myb-interacting proteins and repress transcription of developmentally controlled E2F target genes. Cell 2004, I I 9(2): 18I-193.

8. Morris EJ, Dyson NJ: Retinoblastoma protein partners. Adv Cancer Res 200I, 82: I-54.

9. Lu X, Horvitz HR: lin-35 and lin-53, two genes that antagonize a C. elegans Ras pathway, encode proteins similar to $\mathbf{R b}$ and its binding protein RbAp48. Cell 1998, 95(7):98I-991.

10. Fay DS, Keenan S, Han M: fzr-I and lin-35/Rb function redundantly to control cell proliferation in $\mathrm{C}$. elegans as revealed by a nonbiased synthetic screen. Genes Dev 2002, l 6(4):503-517.

II. Cardoso C, Couillault C, Mignon-Ravix C, Millet A, Ewbank JJ, Fontes M, Pujol N: XNP-I/ATR-X acts with RB, HPI and the NuRD complex during larval development in C. elegans. Dev Biol 2005, 278(I):49-59.

12. Boxem M, van den Heuvel S: lin-35 Rb and cki-I Cip/Kip cooperate in developmental regulation of $\mathrm{GI}$ progression in C. elegans. Development 200I, I 28(2I):4349-4359.

13. Fay DS, Large E, Han M, Darland M: lin-35/Rb and ubc- I8, an E2 ubiquitin-conjugating enzyme, function redundantly to control pharyngeal morphogenesis in C. elegans. Development 2003, I30(14):3319-3330.

14. Fay DS, Qiu X, Large E, Smith CP, Mango S, Johanson BL: The coordinate regulation of pharyngeal development in $\mathrm{C}$. elegans by lin-35/Rb, pha-I, and ubc- 1 8. Dev Biol 2004, 27 I (I): I I-25.

15. Bender AM, Wells O, Fay DS: lin-35/Rb and xnp-I/ATR-X function redundantly to control somatic gonad development in C. elegans. Dev Biol 2004, 273(2):335-349.

16. Cui M, Fay DS, Han M: lin-35/Rb cooperates with the SWI/SNF complex to control Caenorhabditis elegans larval development. Genetics 2004, 167(3): I I77-I I85.

17. Saito RM, Perreault A, Peach B, Satterlee JS, van den Heuvel S: The CDC-14 phosphatase controls developmental cell-cycle arrest in C. elegans. Nat Cell Biol 2004, 6(8):777-783.

18. Reddy KC, Villeneuve AM: C. elegans HIM- 17 links chromatin modification and competence for initiation of meiotic recombination. Cell 2004, I I 8(4):439-452.

19. Grishok A, Sharp PA: Negative regulation of nuclear divisions in Caenorhabditis elegans by retinoblastoma and RNA interference-related genes. Proc Natl Acad Sci USA 2005, 102(48): 17360-17365.

20. Chesney MA, Kidd AR 3rd, Kimble J: gon-I4 functions with class $B$ and class $C$ synthetic multivulva genes to control larva growth in Caenorhabditis elegans. Genetics 2006, 172(2):915-928.

21. Ceol C], Horvitz HR: A new class of C. elegans synMuv genes implicates a Tip60/NuA4-like HAT complex as a negative regulator of Ras signaling. Dev Cell 2004, 6(4):563-576.

22. Boxem M, van den Heuvel S: C. elegans class B synthetic multivulva genes act in $\mathbf{G}(\mathrm{I})$ regulation. Curr Biol 2002, I 2(II):906-9II.

23. Ceol C], Horvitz HR: dpl-I DP and eff-I E2F act with lin-35 Rb to antagonize Ras signaling in C. elegans vulval development. Mol Cell 200I, 7(3):461-73.

24. Lewis PW, Beall EL, Fleischer TC, Georlette D, Link AJ, Botchan MR: Identification of a Drosophila Myb-E2F2/RBF transcriptional repressor complex. Genes Dev 2004, I 8(23):2929-40.

25. Rual JF, Ceron J, Koreth J, Hao T, Nicot AS, Hirozane-Kishikawa T, Vandenhaute J, Orkin SH, Hill DE, van den Heuvel S, et al.: Toward improving Caenorhabditis elegans phenome mapping with an ORFeome-based RNAi library. Genome Res 2004, I4(IOB):2162-2168.

26. Wang D, Kennedy S, Conte D Jr, Kim JK, Gabel HW, Kamath RS, Mello CC, Ruvkun G: Somatic misexpression of germline $\mathbf{P}$ granules and enhanced RNA interference in retinoblastoma pathway mutants. Nature 2005, 436(7050):593-597.

27. Simmer F, Tijsterman M, Parrish S, Koushika SP, Nonet ML, Fire A, Ahringer J, Plasterk RH: Loss of the putative RNA-directed RNA polymerase RRF-3 makes C. elegans hypersensitive to RNAi. Curr Biol 2002, I 2(15): |3|7-1319.

28. Cui M, Kim EB, Han M: Diverse chromatin remodeling genes antagonize the RB-involved SynMuv pathways in C. elegans. Plos Genet 2006, 2(5):e74.

29. Lehner B, Crombie C, Tischler J, Fortunato A, Fraser AG: Systematic mapping of genetic interactions in Caenorhabditis elegans identifies common modifiers of diverse signaling pathways. Nat Genet 2006, 38(8):896-903.

30. Freire Raimundo, van Vugt Marcel ATM, Mamely Ivan, Medema Rene: Claspin: Timing the cell cycle arrest when the genome is damaged. Cell Cycle 2006, 5(24):283I-2834.

31. Ringrose Leonie, Paro Renato: Epigenetic regulation of cellular memory by the Polycomb and Trithorax group proteins. Annu Rev Genet 2004, 38:4I 3-43.

32. Wysocka J, Myers MP, Laherty CD, Eisenman RN, Herr W: Human Sin 3 deacetylase and trithorax-related Set I/Ash2 histone H3-K4 methyltransferase are tethered together selectively by the cell-proliferation factor HCF-I. Genes Dev 2003 , I7(7):896-9|I.

33. Couillault Carole, Pujol Nathalie, Reboul Jérôme, Sabatier Laurence, Guichou Jean-François, Kohara Yuji, Ewbank Jonathan J: TLR-independent control of innate immunity in Caenorhabditis elegans by the TIR domain adaptor protein TIR-I, an ortholog of human SARM. Nature Immunology 2004, 5:488-494.

34. Liberati Nicole T, Fitzgerald Katherine A, Kim Dennis H, Feinbaum Rhonda, Golenbock Douglas T, Ausubel Frederick M: Requirement for a conserved Toll/interleukin-I resistance domain protein in the Caenorhabditis elegans immune response. Proc Nat Acad Sci USA 2004, I 01 (17):6593-6598.

35. Chuang Chiou-Fen, Bargmann Cornelia I: A Toll-interleukin I repeat protein at the synapse specifies asymmetric odorant receptor expression via ASKI MAPKKK signaling. Genes \& Dev 2005, 19:270-28I.

36. Urrutia R: KRAB-containing zinc-finger repressor proteins. Genome Biol 2003, 4(I 0):231.

37. Buyse IM, Shao G, Huang S: The retinoblastoma protein binds to RIZ, a zinc-finger protein that shares an epitope with the adenovirus EIA protein. Proc Natl Acad Sci USA 1995, 92(10):4467-447I

38. Canote R, Du Y, Carling T, Tian F, Peng Z, Huang S: The tumor suppressor gene RIZ in cancer gene therapy (review). Oncol Rep 2002, 9 (I):57-60

39. Skapek SX, Jansen D, Wei TF, McDermott T, Huang W, Olson EN, Lee EY: Cloning and characterization of a novel Kruppel-associated box family transcriptional repressor that interacts with the retinoblastoma gene product, RB. J Biol Chem 2000, 275(10):7212-7223.

40. Greenstein $D$, Hird $S$, Plasterk RH, Andachi $Y$, Kohara $Y$, Wang B, Finney M, Ruvkun G: Targeted mutations in the Caenorhabditis elegans POU homeo box gene ceh- 18 cause defects in oocyte cell cycle arrest, gonad migration, and epidermal differentiation. Genes Dev 1994, 8(16): 1935-1948.

4I. McCarter J, Bartlett B, Dang T, Schedl T: Soma-Germ Cell Interactions in Caenorhabditis elegans: Multiple events of hermaphrodite germline development require the somatic sheat and spermathecal lineages. Dev Biol 1997, I I I: | 2 I- | 43.

42. Myers TR, Greenwald I: lin-35 Rb acts in the major hypodermis to oppose ras-mediated vulval induction in C. elegans. Dev Cell 2005, 8(I): II7-I23.

43. Kimble J, Hirsh D: The postembryonic cell lineages of the hermaphrodite and male gonads in Caenorhabditis elegans. Dev Biol 1979, 70(2):396-4I7.

44. Hall DH, Winfrey VP, Blaeuer G, Hoffman LH, Furuta T, Rose KL, Hobert $O$, Greenstein $D$ : Ultrastructural features of the adult hermaphrodite gonad of Caenorhabditis elegans: relations between the germ line and soma. Dev Biol 1999, 2I $2(1): 10 \mid-123$.

45. Robert VJ, Sijen T, van Wolfswinkel J, Plasterk RH: Chromatin and RNAi factors protect the C. elegans germline against repetitive sequences. Genes Dev 2005, I 9(7):782-787. 
46. Walhout AJ, Sordella R, Lu X, Hartley JL, Temple GF, Brasch MA, Thierry-Mieg N, Vidal M: Protein interaction mapping in C. elegans using proteins involved in vulval development. Science 2000 , 287(5450): I |6-22.

47. Davison EM, Harrison MM, Walhout AJ, Vidal M, Horvitz HR: lin-8, which antagonizes Caenorhabditis elegans Ras-mediated vulval induction, encodes a novel nuclear protein that interacts with the LIN-35 Rb protein. Genetics 2005, I 7 I(3): |0|7-3|

48. Longman D, McGarvey T, McCracken S, Johnstone IL, Blencowe B] Caceres JF: Multiple interactions between SRmI60 and SR family proteins in enhancer-dependent splicing and development of C. elegans. Curr Biol 200 I, I I (24): | 923-1933.

49. Barbee SA, Lublin AL, Evans TC: A novel function for the Sm proteins in germ granule localization during $\mathbf{C}$. elegans embryogenesis. Curr Biol 2002, I 2(I 7): I502-I506.

50. Cui M, Chen J, Myers TR, Hwang BJ, Sternberg PW, Greenwald I, Han $M$ : SynMuv genes redundantly inhibit lin-3/EGF expression to prevent inappropriate vulval induction in C. elegans. Dev Cell 2006, I 0(5):667-672.

5I. Poulin G, Dong Y, Fraser AG, Hopper NA, Ahringer J: Chromatin regulation and sumoylation in the inhibition of Ras-induced vulval development in Caenorhabditis elegans. Embo J 2005 24( I 4):26|3-2623.

52. Barbee SA, Evans TC: The Sm proteins regulate germ cell specification during early C. elegans embryogenesis. Dev Biol 2006 , 29 I(I): |32-143.

53. Shet U, Parker R: Decapping and decay of messenger RNA occur in cytoplasmic processing bodies. Science 2003 300(5620):805-8.

54. Ingelfinger D, Arndt-Jovin DJ, Luhrmann R, Achsel T: The human LSmI-7 proteins colocalize with the mRNA-degrading enzymes Dcpl/2 and Xrnl in distinct cytoplasmic foci. Rna 2002, 8( I 2): | 489-| $50 \mid$.

55. Ding L, Spencer A, Morita K, Han M: The developmental timing regulator AIN-I interacts with miRISCs and may target the argonaute protein ALG-I to cytoplasmic P bodies in C. elegans. Mol Cell 2005, I 9(4):437-47.

56. Liu J, Rivas FV, Wohlschlegel J, Yates JR 3rd, Parker R, Hannon GJ: A role for the P-body component GWI82 in microRNA function. Nat Cell Biol 2005, 7(I2): |26|-6.

57. Pillai RS, Bhattacharyya SN, Artus CG, Zoller T, Cougot N, Basyuk E, Bertrand E, Filipowicz W: Inhibition of translational initiation by Let-7 MicroRNA in human cells. Science 2005 309(5740): I573-6.

58. Kim JK, Gabel HW, Kamath RS, Tewari M, Pasquinelli A, Rual JF, Kennedy S, Dybbs M, Bertin N, Kaplan JM, et al.: Functional genomic analysis of RNA interference in C. elegans. Science 2005, 308(5725): I 164- II67.

59. Duchaine TF, Wohlschlegel JA, Kennedy S, Bei Y, Conte D Jr, Pang K, Brownell DR, Harding S, Mitani S, Ruvkun G, et al:: Functional proteomics reveals the biochemical niche of $C$. elegans DCR-I in multiple small-RNA-mediated pathways. Cell 2006, I 24(2):343-354

60. Chang W, Tilmann C, Thoemke K, Markussen FH, Mathies LD, Kimble J, Zarkower D: A forkhead protein controls sexual identity of the C. elegans male somatic gonad. Development 2004 I 3 |(6): | 425- | 436.

61. Dupuy D, Li Q, Deplancke B, Boxem M, Hao T, Lamesch P, Sequerra $\mathrm{R}$, Bosak S, Doucette-Stamm L, IA H, et al:: A First version of the Caenorhabditis elegans Promoterome. Genome Res 2004, 14:2169-2175

62. Kennedy S, Wang D, Ruvkun G: A conserved siRNA-degrading RNase negatively regulates RNA interference in C. elegans. Nature 2004, 427(6975):645-649.

63. Simmer F, Moorman C, van der Linden AM, Kuijk E, van den Berghe PV, Kamath RS, Fraser AG, Ahringer J, Plasterk RH: Genome-wide RNAi of $C$. elegans using the hypersensitive rrf-3 strain reveals novel gene functions. PLoS Biol 2003, I(I):EI2.

64. Kamath RS, Fraser AG, Dong Y, Poulin G, Durbin R, Gotta M, Kanapin A, Le Bot N, Moreno S, Sohrmann M, et al.: Systematic functional analysis of the Caenorhabditis elegans genome using RNAi. Nature 2003, 42 I(6920):23I-237.

65. Lehner B, Calixto A, Crombie C, Tischler J, Fortunato A, Chalfie M, Fraser A: Loss of LIN-35, the Caenorhabditis elegans ortholog of the tumor suppressor pl05Rb, results in enhanced RNA interference. Genome Biol 2006, 7(I):R4
66. The biology and geneome of $\mathbf{C}$. elegans database [http:// www.wormbase.org]

67. Promoterome database [http://vidal.dfci.harvard.edu/promoter omedb/]
Publish with Bio Med Central and every scientist can read your work free of charge

"BioMed Central will be the most significant development for disseminating the results of biomedical research in our lifetime. "

Sir Paul Nurse, Cancer Research UK

Your research papers will be:

- available free of charge to the entire biomedical community

- peer reviewed and published immediately upon acceptance

- cited in PubMed and archived on PubMed Central

- yours - you keep the copyright
BioMedcentral 